\title{
Application of Quantitative Structure-Retention Relationships for Reversed-Phase Liquid Chromatographic Separation of Pesticides ${ }^{\dagger}$
}

\author{
Ho Seob KIM and Dai Woon LEE ${ }^{\dagger}$ \\ Department of Chemistry, Yonsei University, Seoul 120-749, Korea
}

\begin{abstract}
Quantitative structure-retention relationships (QSRRs) models that predict the reversed-phase liquid chromatographic retention behavior of carboxamides and oxadiazoles are proposed. The intermolecular interaction, isomeric effect and substituent effect were explained by the descriptors determined from calculations with MM+ and AM1 methods. The retention of carboxamides was elucidated by using solvent-accessible surface area and $\mathrm{x}$ component of dipole moment. For oxadiazoles, 1-octanol/water partition coefficient $(\log P)$ and dipole moment were useful descriptors.
\end{abstract}

Keywords Quantitative structure-retention relationship, reversed-phase liquid chromatography, carboxamides, oxadiazoles, MM+ calculation, AM1 calculation

The chromatographic retention results from intermolecular interactions such as solute-stationary phase, solute-mobile phase and mobile phase-stationary phase. Since chromatographic conditions may be kept constant in a separation, solute is the only variable component in a chromatographic system. ${ }^{1}$

Quantitative structure-retention relationships (QSRRs) are the methodology of relating chemical structures of solute with chromatographic retention parameters such as capacity factor $\left(k^{\prime}\right)$. QSRRs approach has been used to study the properties of solute affecting the retention. There are many available descriptors, such as topological, geometrical, electronic, physicochemical and intermolecular descriptors, commonly used in QSRRs studies. ${ }^{1,2}$

It has been difficult to describe the structural effect of solute on retention due to the unsuitableness of descriptors. But molecular mechanics and quantum chemical methods allow us to determine the structurally related descriptors easily. If retention data were dependent on the molecular structure, QSRRs studies are suitable for testing new structurally related descriptors and hence QSRRs methods may be useful to discern a new method of representing chemical structures.

In this study, we attempted to apply the molecular mechanics and quantum chemical calculations to QSRRs study in order to find structurally related descriptors and to explain the retention behavior of carboxamides and oxadiazoles.

$\dagger$ Presented at the ASIANALYSIS III, August $20-24,1995$, Seoul, Korea.

tt To whom correspondence should be addressed.

\section{Experimental}

\section{Reagents and chemicals}

Eight carboxamides and seven oxadiazoles ${ }^{3}$ were used for this study (Figs. 1 and 2); their retention data are listed in Tables 1 and 2.

Methanol (HPLC grade) and acetonitrile (HPLC grade) were purchased from J. T. Baker (Phillipsburg, NJ, USA). Water was purified by using a Milli-Q water purification system (Millipore, Bedford, MA, USA).

\section{Procedure}

All chromatograms were obtained by using a Shimadzu LC-10AD liquid chromatograph (Kyoto, Japan) equipped with a Shimadzu spectrophotometric detector SPD-10A at a wavelength of $254 \mathrm{~nm}$. An Alltech NucleosilC1 8 column $(250 \times 4.6 \mathrm{~mm}$ i.d., Deerfield, IL, USA) was used. Isocratic elution was carried out with the mobile phases which were proper for deter-

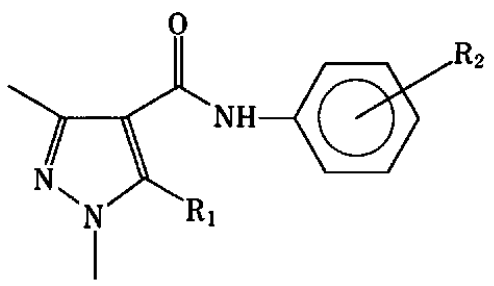

\begin{tabular}{ccccccccc}
\hline No. & 1 & 2 & 3 & 4 & 5 & 6 & 7 & 8 \\
\hline $\mathbf{R}_{1}$ & $\mathrm{CH}_{3}$ & $\mathrm{CH}_{3}$ & $\mathrm{Cl}$ & $\mathrm{CH}_{3}$ & $\mathrm{CH}_{3}$ & $\mathrm{CH}_{3}$ & $\mathrm{Cl}$ & $\mathrm{Cl}$ \\
$\mathrm{R}_{2}$ & $p-\mathrm{OCH}_{3}$ & $m-\mathrm{OCH}_{3}$ & $m-\mathrm{OCH}_{3}$ & $p-\mathrm{Cl}$ & $o-0 \mathrm{OCH}_{3}$ & $o-\mathrm{Cl}$ & $p-\mathrm{Cl}$ & $o-0 \mathrm{CH}_{3}$ \\
\hline
\end{tabular}

Fig. 1 Structures of carboxamides. 


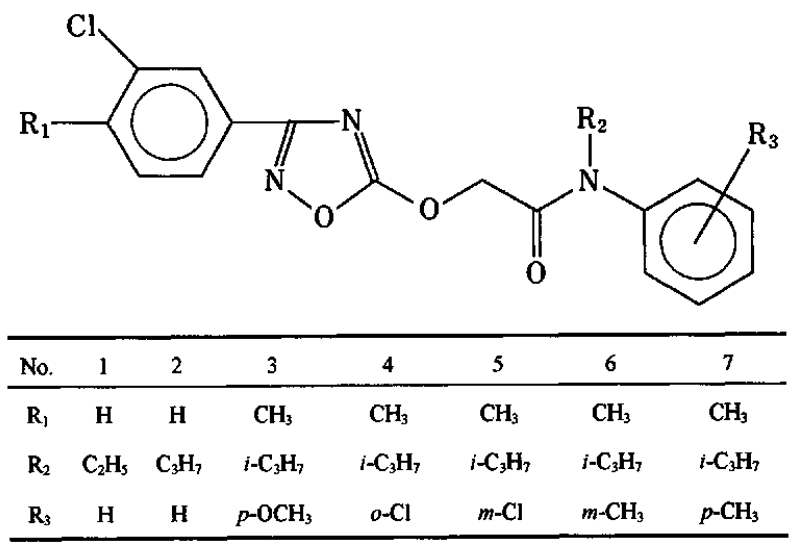

Fig. 2 Structures of oxadiazoles.

Table 1 Capacity factors $\left(\log k^{\prime}\right)$ of carboxamides at $70 \%$ $\mathrm{MeOH}-\mathrm{H}_{2} \mathrm{O}$ and $50 \% \mathrm{MeCN}-\mathrm{H}_{2} \mathrm{O}$

\begin{tabular}{ccc}
\hline No. of solute & $\begin{array}{c}\log k^{\prime} \text { at } 70 \% \\
\mathrm{MeOH}-\mathrm{H}_{2} \mathrm{O}\end{array}$ & $\begin{array}{c}\log k^{\prime} \text { at } 50 \% \\
\mathrm{MeCN}-\mathrm{H}_{2} \mathrm{O}\end{array}$ \\
\hline 1 & -0.734 & -0.211 \\
2 & -0.528 & -0.020 \\
3 & -0.151 & 0.482 \\
4 & 0.157 & 0.631 \\
5 & 0.344 & 0.647 \\
6 & 0.489 & 0.896 \\
7 & 0.482 & 1.092 \\
8 & 0.798 & 1.188 \\
\hline
\end{tabular}

Table 2 Capacity factors $\left(\log k^{\prime}\right)$ of oxadiazoles at $70 \%$ $\mathrm{MeOH}-\mathrm{H}_{2} \mathrm{O}$ and $80 \% \mathrm{MeCN}-\mathrm{H}_{2} \mathrm{O}$

\begin{tabular}{ccc}
\hline No. of solute & $\begin{array}{c}\log k^{\prime} \text { at } 70 \% \\
\mathrm{MeOH}-\mathrm{H}_{2} \mathrm{O}\end{array}$ & $\begin{array}{c}\log k^{\prime} \text { at } 80 \% \\
\mathrm{MeCN}-\mathrm{H}_{2} \mathrm{O}\end{array}$ \\
\hline 1 & 1.625 & 0.140 \\
2 & 1.977 & 0.358 \\
3 & 2.500 & 0588 \\
4 & 2.687 & 0.859 \\
5 & 2.770 & 0.829 \\
6 & 2.814 & 0.908 \\
7 & 2.868 & 0.928 \\
\hline
\end{tabular}

mining descriptors. They are $70 \%$ methanol-water and $50 \%$ acetonitrile-water for carboxamides, and $70 \%$ methanol-water and $80 \%$ acetonitrile-water for oxadiazoles, respectively. The flow rate was fixed at $1.0 \mathrm{ml} /$ min. The retention times for all solutes were measured three times at $35^{\circ} \mathrm{C}$ and their average values were used for the calculation of the capacity factors $\left(k^{\prime}\right)$. The retention time of the solvent was used as $t_{0}$ value.

\section{Descriptor generation}

Calculations for getting descriptors were carried out with an IBM compatible PC using HyperChem (Hypercube, Waterloo, Ontario, Canada). The geometry optimization calculation of each solute was carried out by $\mathrm{MM}+{ }^{4}$ in a periodic box. Total energy and the energy of each individual bonding component (bonds, angles, dihedrals, van der Waals interactions, hydrogen bonding, electrostatic energy) obtained from optimization calculation were used as descriptors. Using optimized geometry, single point calculations were done by a semi-empirical method, AM1.5,6 Then total energy in semi-empirical calculation, the energy components, heat of formation, dipole moment, and components of dipole moment were used as descriptors. Solvent-accessible surface area, solvent-accessible molecular volume, 1-octanol/water partition coefficient $(\log P)$, molar refractivity, and polarizability were also calculated with ChemPlus module (Hypercube, Waterloo, Ontario, Canada).

\section{Regression analysis}

Multiple linear regression analysis ${ }^{7}$ was used for descriptor selection and model construction. Preliminary information on the interrelationships among the descriptors was obtained from the correlation matrix. Also, multicollinearity was examined using tolerance. Multiple linear regression analysis was performed by a stepwise procedure, followed by model generation. The criteria for judging the best model were multiple correlation coefficient, standard error, and overall Fvalue for analysis of variance. Statistical calculations were performed with Macintosh Classic II using SYSTAT software (SYSTAT, Evanston, IL, USA).

\section{Results and Discussion}

One of the main goals of QSRRs studies is to determine the most informative structural descriptors which can elucidate the retention behavior in a given chromatographic system. In the previous work ${ }^{2}$, the retention of carboxamides and oxadiazoles could not be explained by intermolecular interaction based on the group contribution due to isomeric effects. But, in this study, descriptors including isomeric effect and substituent effect were obtained from molecular structure optimized by molecular mechanics calculations.

The best models selected for carboxamides and oxadiazoles are represented in Table 3 to Table 6, respectively. Plots of the observed $v s$. calculated retention value for carboxamides and oxadiazoles are shown in Figs. 3 and 4, respectively. They showed good correlation.

The standardized coefficients of the selected descriptors were directly compared in order to determine the relative contribution of each descriptor to the retention value. Descriptors which have larger magnitude of the standardized coefficient would make a greater contribution to the retention. Therefore, solvent-accessible surface area had a major effect on the retention of carboxamides (Tables 3 and 4). Since the sign of the standardized coefficient of solvent-accessible surface area was negative, the retention value of solute that has a 
Table 3 Regression model developed for the carboxamides eluted with $70 \% \mathrm{MeOH}-\mathrm{H}_{2} \mathrm{O}$

\begin{tabular}{|c|c|c|c|}
\hline Std. coefficient & Std. error & Tolerance & Descriptor \\
\hline-0.542 & 0.005 & 0.420 & $\begin{array}{l}\text { solvent-accessible } \\
\text { surface area }\end{array}$ \\
\hline-0.540 & 0.044 & 0.551 & $\begin{array}{l}x \text { component of } \\
\text { dipole moment }\end{array}$ \\
\hline 0.442 & 0.009 & 0.363 & total energy of $\mathrm{MM}+$ \\
\hline$R_{\text {Adj }}^{2}=0.976$ & \multicolumn{2}{|c|}{ Std. error $=0.097$} & $N=8 \quad F=70.067$ \\
\hline
\end{tabular}

Table 4 Regression model developed for the carboxamides eluted with $50 \% \mathrm{MeCN}-\mathrm{H}_{2} \mathrm{O}$

\begin{tabular}{|c|c|c|c|}
\hline Std. coefficient & Std. error & Tolerance & Descriptor \\
\hline-0.883 & 0.004 & 0.958 & $\begin{array}{l}\text { solvent-accessible } \\
\text { surface area }\end{array}$ \\
\hline-0.197 & 0.041 & 0.896 & $\begin{array}{l}x \text { component of } \\
\text { dipole moment }\end{array}$ \\
\hline-0.292 & 0.000 & 0.924 & $\begin{array}{l}\text { isolated atomic } \\
\text { energy of } \mathrm{AM} 1\end{array}$ \\
\hline$R_{\mathrm{Adj}}^{2}=0.948$ & \multicolumn{2}{|c|}{ Std. error $=0.113$} & $N=8 \quad F=43.762$ \\
\hline
\end{tabular}

Table 5 Regression model developed for the oxadiazoles eluted with $70 \% \mathrm{MeOH}-\mathrm{H}_{2} \mathrm{O}$

\begin{tabular}{cccc}
\hline Std. coefficient & Std. error & Tolerance & Descriptor \\
\hline 0.821 & 0.043 & 0.837 & $\log P$ \\
0.322 & 0.021 & 0.837 & dipole moment \\
$R_{\text {Adj }}^{2}=0.986$ & Std. error $=0.056$ & $N=7 \quad F=220.038$ \\
\hline
\end{tabular}

Table 6 Regression model developed for the oxadiazoles eluted with $80 \% \mathrm{MeCN}-\mathrm{H}_{2} \mathrm{O}$

\begin{tabular}{cccc}
\hline Std. coefficient & Std. error & Tolerance & Descriptor \\
\hline 0.911 & 0.038 & 0.837 & $\log P$ \\
0.170 & 0.018 & 0.837 & dipole moment \\
$R_{\text {Adj }}^{2}=0.976$ & Std. error $=0.048$ & $N=7 F=121.117$ \\
\hline
\end{tabular}

larger solvent-accessible surface area would be smaller. It can be seen from the plots of the solvent-accessible surface area $v s$. retention value for carboxamides (Fig. 5).

Basically, two types of interaction between solute and solvated stationary phase surface can be considered. ${ }^{8}$ First, the solute can interact with the adsorbed solvent layer and then remain on the layer. This interaction will take place when the interactions between the solute and the stationary phase are relatively weak compared with interaction between the solvent and the stationary phase. This has been known as sorption interaction. Second, the solute can displace the solvent molecules from the stationary phase surface and interact directly with the surface. This displacement interaction occurs when the interaction between the solute and the stationary phase is
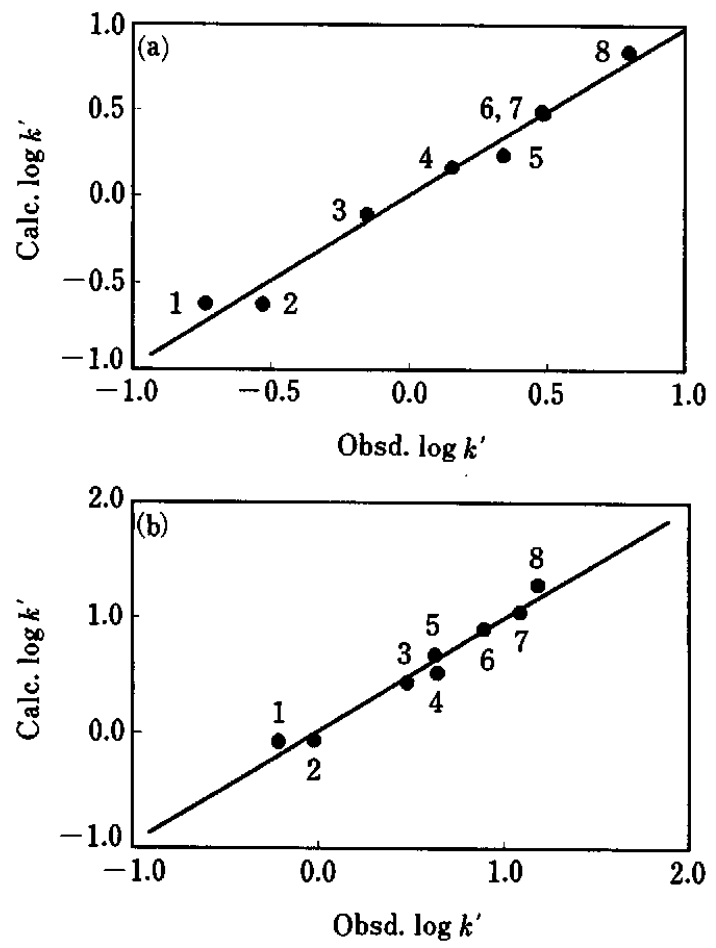

Fig. 3 Plots of calculated $\log k^{\prime} v s$. observed $\log k^{\prime}$ for carboxamides at (a) $70 \% \mathrm{MeOH}-\mathrm{H}_{2} \mathrm{O}$ and (b) $50 \% \mathrm{MeCN}-$ $\mathrm{H}_{2} \mathrm{O}$.
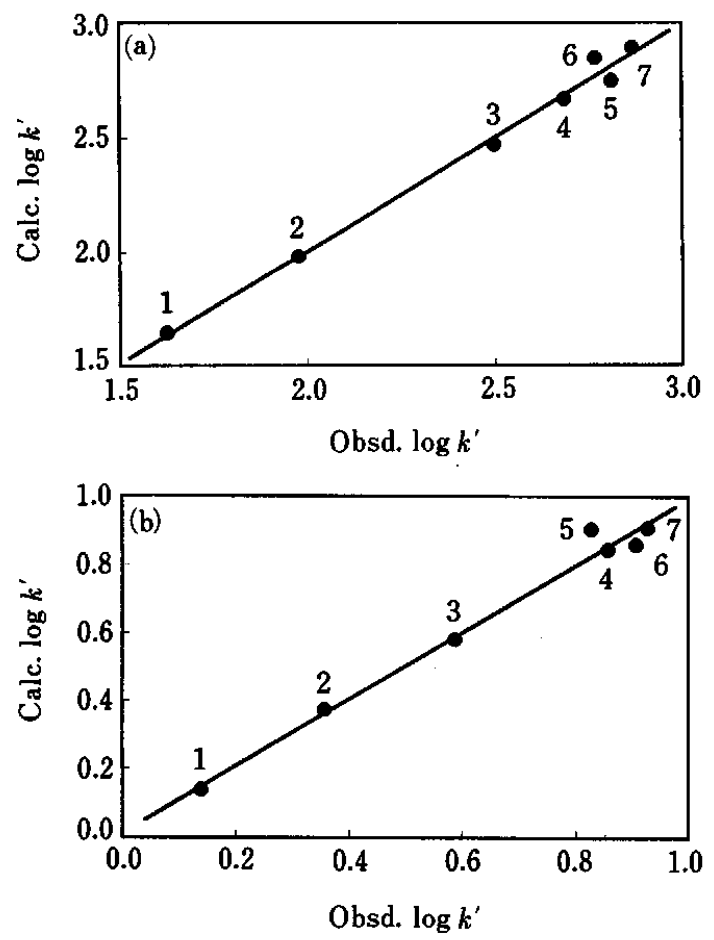

Fig. 4 Plots of calculated $\log k^{\prime} v s$. observed $\log k^{\prime}$ for oxadiazoles at (a) $70 \% \mathrm{MeOH}-\mathrm{H}_{2} \mathrm{O}$ and (b) $80 \% \mathrm{MeCN}-$ $\mathrm{H}_{2} \mathrm{O}$. 

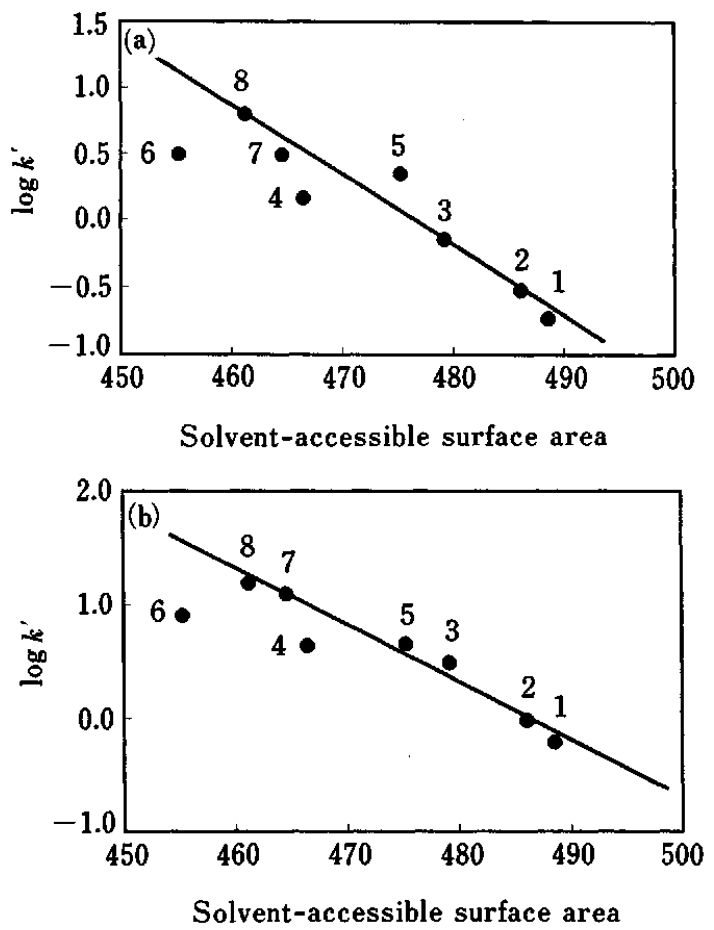

Fig. 5 Plots of $\log k^{\prime} v$ s. solvent-accessible surface area $\left(\AA^{2}\right)$ for carboxamides at (a) $70 \% \mathrm{MeOH}-\mathrm{H}_{2} \mathrm{O}$ and (b) $50 \%$ $\mathrm{MeCN}-\mathrm{H}_{2} \mathrm{O}$.

much stronger than interaction between the solvent and the stationary phase.

If there are multi-layers of solvent on the stationary phase surface and the solute interacts with lower layer of solvent by displacing solvent from upper layer, the cavity formation energy in solvent layer increases with the surface area of the solute and hence retention of the solute decreases as the surface area increases. Therefore, from the relationship between solvent-accessible surface area and retention, it was supposed that the major interaction affecting the retention of carboxamides was a displacement interaction.

In Fig. 5, some solutes, specially carboxamides \#4 and \#6 showed high discrepancy. It means that there was a different effect on the retention of carboxamides \#4 and \#6 compared with that on other carboxamides. Multiple correlation coefficient, except carboxamide \#4 and $\# 6$, was 0.940 at $70 \%$ methanol and 0.976 at $50 \%$ acetonitrile (Fig. 5). Other carboxamides have $\mathrm{OCH}_{3}$ at $\mathrm{R}_{2}$, while carboxamides $\# 4$ and $\# 6$ have $\mathrm{Cl}$ at $\mathrm{R}_{2}$. Charge density of atoms in mono-substituted benzenes was calculated by AM1 in order to investigate the interrelation between charge density and retention. Since substituents could be divided into three groups based on the relationship between charge density of substituent and retention (Fig. 6), it could be assumed that the substituents which belonged to a different group had a different effect on electronic distribution in molecules. Since $\mathrm{OCH}_{3}$ and $\mathrm{Cl}$ belonged to different groups, they might separately have different effects on the electronic distribution in the molecule and on the
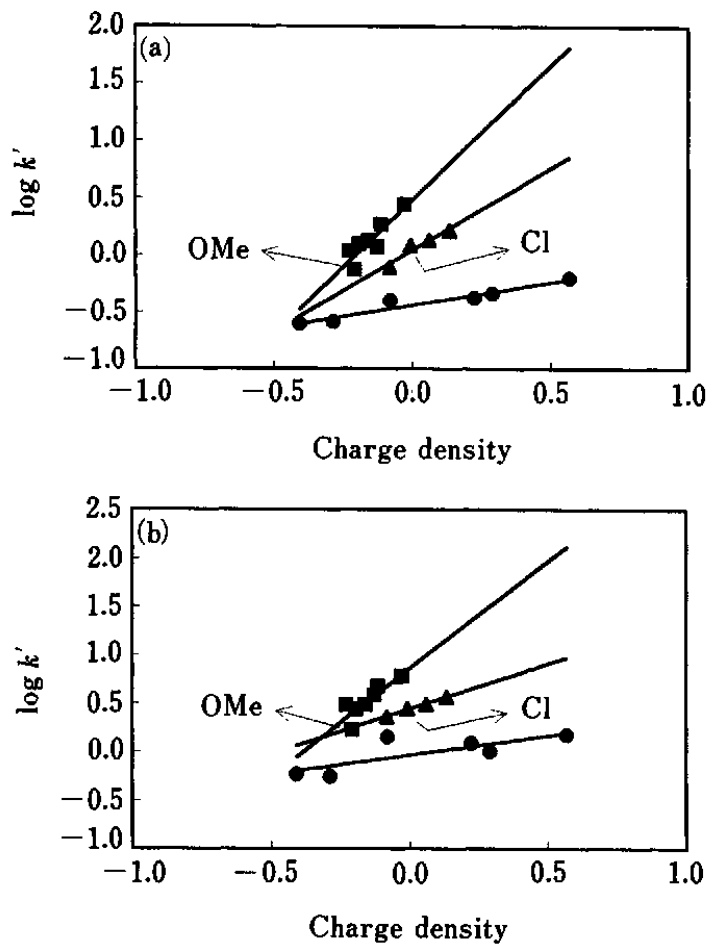

Fig. 6 Plots of $\log k^{\prime} v s$. charge density of the first atom of substituent for 1-substituted benzenes at (a) $70 \% \mathrm{MeOH}-$ $\mathrm{H}_{2} \mathrm{O}$ and (b) $50 \% \mathrm{MeCN}-\mathrm{H}_{2} \mathrm{O}$.

retention ultimately.

The solvent-accessible surface area reflects the ability of a solute to take part in a specific dispersive interaction described by a displacement interaction. The $\mathrm{x}$ component of dipole moment was assumed to reflect the electronic effect of substituent. Also, bulkiness related with descriptors such as total energy and isolated atomic energy was applied to improve the predicting ability of the regression model (Tables 3 and 4).

Comparing the magnitude of standardized coefficients, one could conclude that $\log P$ had a major effect on the retention for oxadiazoles (Tables 5 and 6). The $\log P$ has served as a measure of hydrophobicity and the interrelation between $\log P$ and retention in reversedphase chromatographic system has been studied in large numbers. The $\log P$ reflects the ability of oxadiazoles to participate in nonspecific dispersive interactions in the chromatographic system. Plots of $\log k^{\prime} v s . \log P$ for oxadiazoles are shown in Fig. 7. Oxadiazoles \#3, \#6 and $\# 7$ which have $\mathrm{OCH}_{3}$ or $\mathrm{CH}_{3}$ at $\mathrm{R}_{3}$ and oxadiazoles \#4 and \#5 having $\mathrm{Cl}$ at $\mathrm{R}_{3}$ showed different deviations from a straight line according to the substituent. In Fig. $6, \mathrm{OCH}_{3}$ and $\mathrm{CH}_{3}$ belonged to the same group, while $\mathrm{Cl}$ belonged to another group. Therefore, their electronic effect on retention might be different. The dipole moment was assumed to reflect the electronic effect of each substituent.

It has been considered that there are two interactions which play a role in solute retention in RPLC, dispersive interaction and electrostatic interaction. Dispersive 

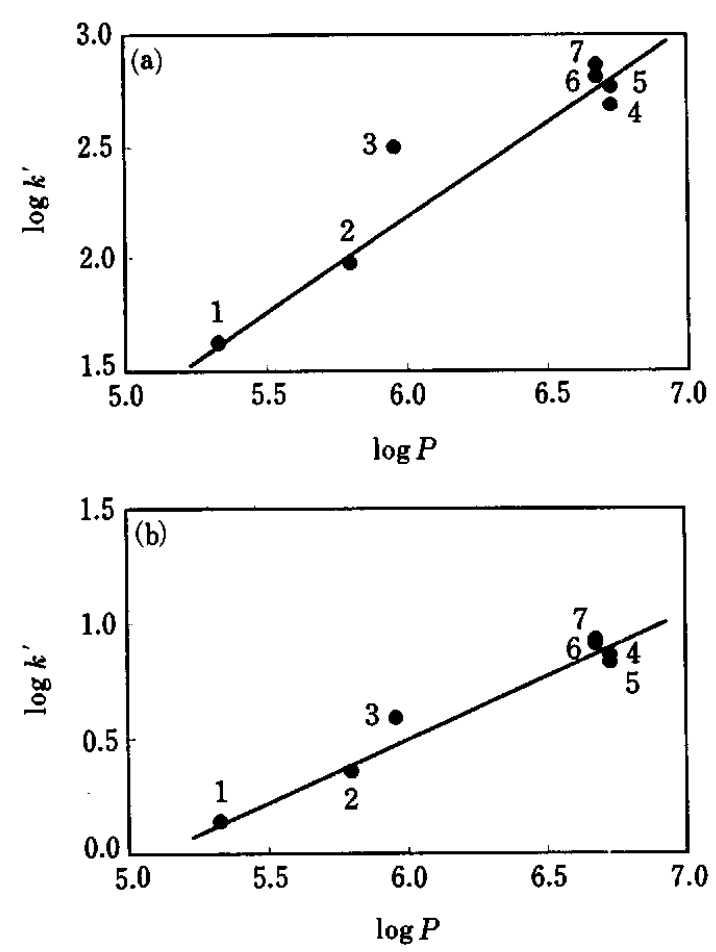

Fig. 7 Plots of $\log k^{\prime}$ vs. $\log P$ for oxadiazoles at (a) $70 \%$ $\mathrm{MeOH}-\mathrm{H}_{2} \mathrm{O}$ and (b) $80 \% \mathrm{MeCN}-\mathrm{H}_{2} \mathrm{O}$.

interaction might be considered between nonpolar groups of solute and nonpolar stationary phase. On the other hand, electrostatic interaction would be expected between polar groups of solute and mobile phase. The regression models for carboxamides and oxadiazoles included both dispersive descriptors, such as solventaccessible surface area and $\log P$, and electrostatic descriptors such as dipole moment. Of course, their contribution to the retention would be different. Therefore, the driving force of the retention of carboxamides and oxadiazoles would be the combination of dispersive and electrostatic interactions. We have obtained the same results in the study of quinolones. ${ }^{9}$

This study was supported by the Center for Molecular Catalysis and the Korea Science and Engineering Foundation. We thank Mr. Hoon Joo Kim of Lucky Research Park for his assistance in the application of the SYSTAT software.

\section{References}

1. R. Kaliszan, J. Chromatogr. A, 656, 417 (1993).

2. H. S. Kim, T. K. Kim and D. W. Lee, J. Liq. Chromatogr., 17, 2615 (1994).

3. Carboxamides are obtained from Dr. Yong Whan Kim and oxadiazoles are obtained from Korea Research Institute of Chemistry and Technology.

4. Modified MM2 [N. L. Allinger, J. Am. Chem. Soc., 99, 8127 (1977).]

5. M. J. S. Dewar, E. G. Zoebisch, E. F. Healy and J. J. P. Stewart, J. Am. Chem. Soc., 107, 3902 (1985).

6. M. J. S. Dewar and K. M. Dieter, J. Am. Chem. Soc., 108, 8075 (1986).

7. A. A. Afifi and V. Clark, "Computer-Aided Multivariate Analysis", Van Nostrand Reinhold Co., New York, 1990.

8. R. P. W. Scott, "Silica Gel and Bonded Phases", Wiley, New York, 1993.

9. H. S. Kim and D. W. Lee, J. Chromatogr. A, 722, 69 (1996).

(Received October 19, 1995)

(Accepted January 8, 1996) 